\title{
Orbital floor reconstruction with polypropylene mesh and conchal cartilage composite graft: A novel technique
}

Ravi Sachidananda MS, DNB, MRCS, DOHNS, FRCS (ORL-HNS), Satish Kumaran MDS, MFDRCS, Srividya BDS, Sandeep Dachuri MS, DNB Integrated Head and Neck Services People Tree Hospitals, Bangalore, India drravi@peopletreehospitals.com

Orbital fractures are common facial fractures in the Indian subcontinent. They occur in either isolation or a part of pan facial fractures. Repairing the fracture is paramount to restore the continuity of the orbital floor, support the orbital contents, prevent fibrosis and prevent herniation of the orbital contents into the maxillary or ethmoidal sinuses. Inadequate treatment leads to functional impairment like atrophy of orbital tissues, enophthalmos, and diplopia.

Commonly, the patient sustains multiple fractures and therefore simple reduction may not be an option. An implant is therefore necessary to reconstruct the floor of the orbit. Although many materials are available to reconstruct the orbital floor, including autologous and synthetic material there is no consensus regarding the ideal material. Autologous materials are readily available material like cartilage (auricular or septal) or bone (ilium, rib, and calvarium). The bone grafts have the advantage of being stable and strong, however they are associated with distant donor site morbidity and postoperative complications. Further, stabilisation is sometimes an issue especially in large comminuted fractures where there is need of pliability for adaptation into the orbital socket.

The aim of the study was to evaluate the role of composite graft with polypropylene mesh and conchal cartilage in orbital floor reconstruction.

\section{Methods}

A retrospective chart review of 16 (2014-2017) patients who presented with maxillary and orbital fractures was conducted. Each patient was evaluated for the presence of enophthalmos, diplopia, anaesthesia or paraesthesia of the infraorbital nerve, and restriction of ocular motility. Diplopia was evaluated in each gaze (vertical and horizontal). Muscular entrapment was assessed using forced duction test under anaesthesia. A CT scan of Head and Facial bones were done in all the planes with $3 \mathrm{D}$ reconstruction. Herniation and entrapment of orbital soft tissue and the inferior rectus muscle were evaluated on midfacial coronal and axial CT.

Operative technique

Conchal cartilage harvest was done by either posterior or anterior approach. Local anaesthesia was infiltrated and a skin flap was raised. Entire conchal cartilage was harvested and the wound was closed with a drain. Pan facial fractures were fixed with centripetal approach moving from stable to unstable zone. Access to the orbital floor was through the laceration or a subcilliary incision. Once the entire orbital floor defect had been explored and fracture reduced, the forced duction test was done to check for muscle entrapment. The graft size was estimated. A synthetic polypropylene mesh was wrapped around the conchal graft with prolene sutures. The fracture was again exposed with a retractor and the herniated fat repositioned. Three patients required endoscopic assistance through Caldwell luc approach for fracture reduction and graft stabilisation. The composite graft was slowly inserted into the orbital floor to ensure complete seal and stability. All grafts were placed on the orbital floor and the mesh fixed anteriorly to the infraorbital plate. Any excess material was trimmed. The patients were discharged on day 2 or 3 and were postoperatively monitored for visual acuity, mobility and any orbital bleed. During the inpatient stay, patients were treated with steroid and antibiotics.

Each patient was followed up at 10 days, 1 month, and 3-6months.
Results:

None of the 16 patients had significant complications. Postoperative visual acuity matched to the preoperative levels in all the patients. There was no diplopia. Mild ectropion was noted in 2 patients and scleral show in 3 patients. No extrusion or donor site morbidity was noted

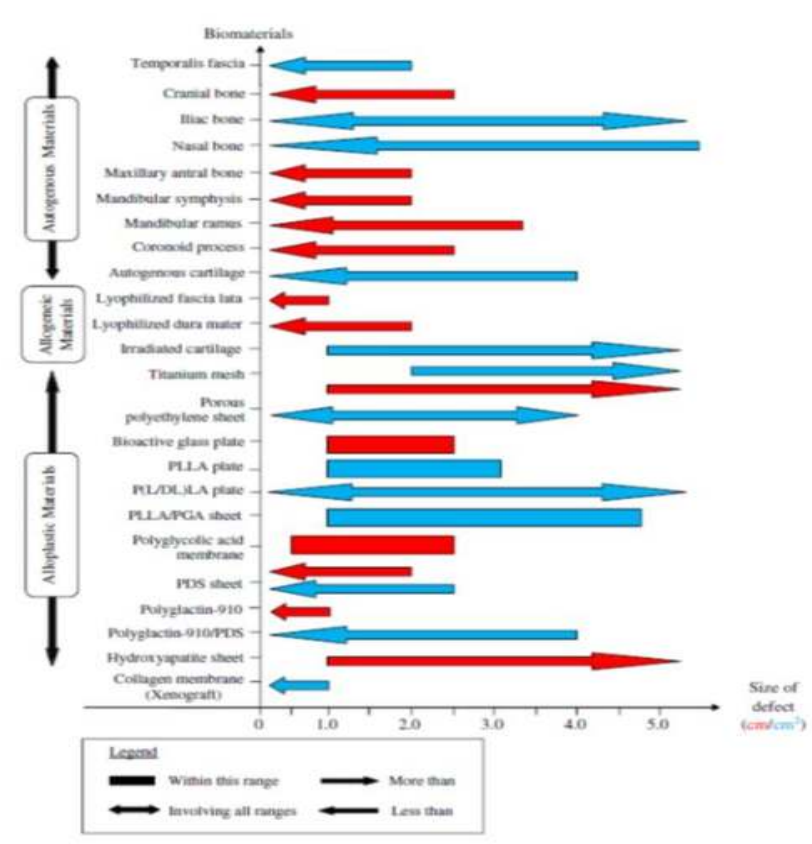

Choice of material vs defect size(Gunarajah et al)

Discussion

Almost 20 different materials are available to reconstruct the orbital floor fractures. They can be classified into autologus, allogenic and synthetic (1) (2). A systematic review demonstrated the utility and choice based on defect size. (Table). A perfect biomaterial would have to be biofriendly, nonallergic and noncarcinogenic. It should also be cost effective, readily available and easy to handle. The success of a graft material depends on the type of material design, biocompatibility and the choice of surgical technique. Whenever an autogenous cartilage graft is preferred, both conchal and nasal septal cartilage provide grafts with minimum morbidity at the donor site (3) The advantage of conchal cartilage is its smooth adaptation on to the orbital floor. The main disadvantage of this technique is the donor site scar and reinforcement needs in large defects.

Wrapping this with pliable polypropylene mesh helps in contouring the graft and helps to give the extra length in large defects. Further, the net like mesh helps to stabilise the composite graft to the infraorbital plate so that no displacement takes place. The main disadvantage of this technique as we see it is the extra time required to harvest from donor site and the time needed to stabilise the graft in an accurate position in large defects where stability and pliability of the graft has to be balanced in graft placement to get optimal results.

Conclusion:

Conchal cartilage and polypropylene mesh is a reliable and novel technique for the repair of orbital floor defects.

References

1. Avashia YJ, Sastry A, Fan KL, Mir HS, Thaller SR. Materials used for reconstruction after orbital floor fracture. J Craniofac Surg. 2012 Nov;23(7 Suppl 1):1991-7.

2. Gunarajah DR, Samman N. Biomaterials for repair of orbital floor blowout fractures: a systematic review. J Oral Maxillofac Surg Off J Am Assoc Oral Maxillofac Surg. 2013 Mar;71(3):550-70.

3. Bayat M, Momen-Heravi F, Khalilzadeh O, Mirhosseni Z, Sadeghi-Tari A. Comparison of conchal cartilage graft with nasal septal cartilage graft for reconstruction of orbital floor blowout fractures. Br J Oral Maxillofac Surg. 2010 Dec;48(8):617-20.

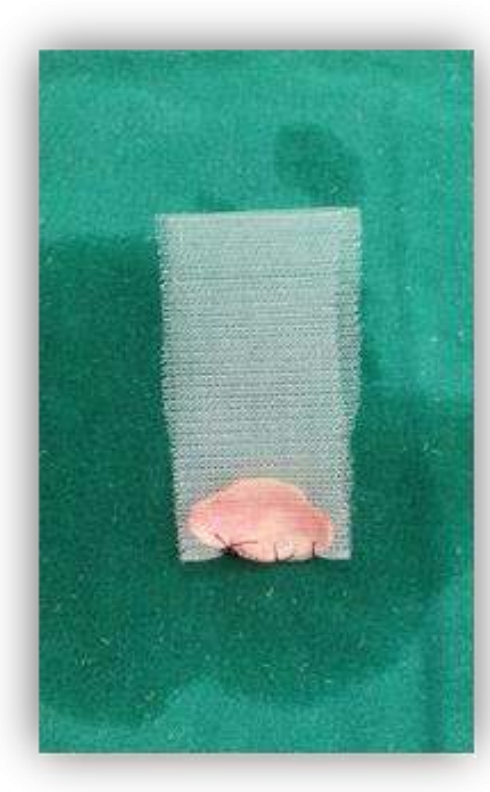

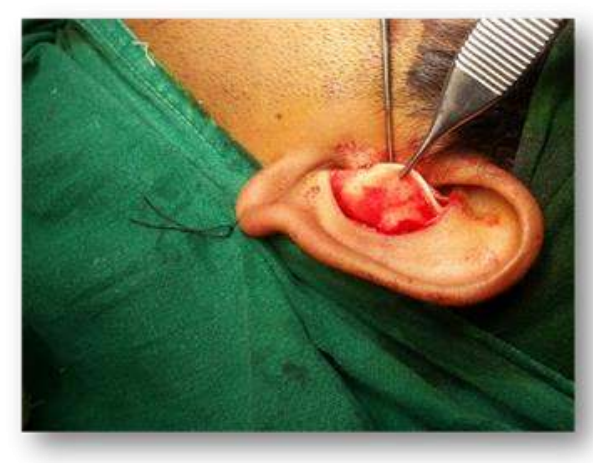
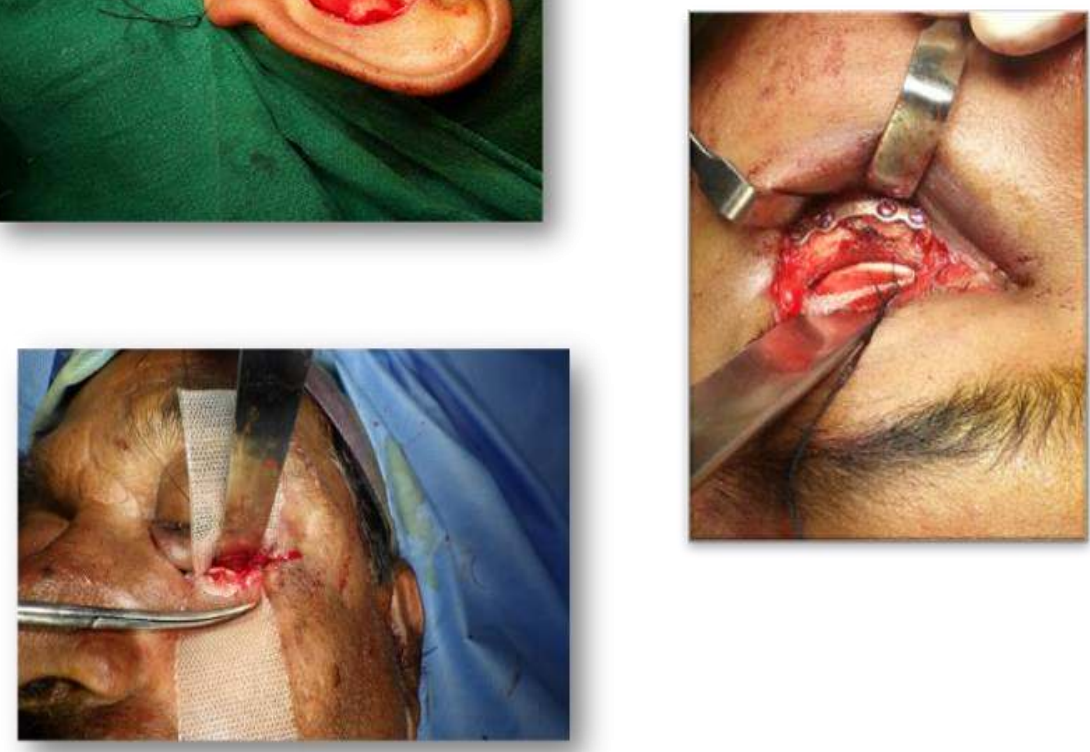

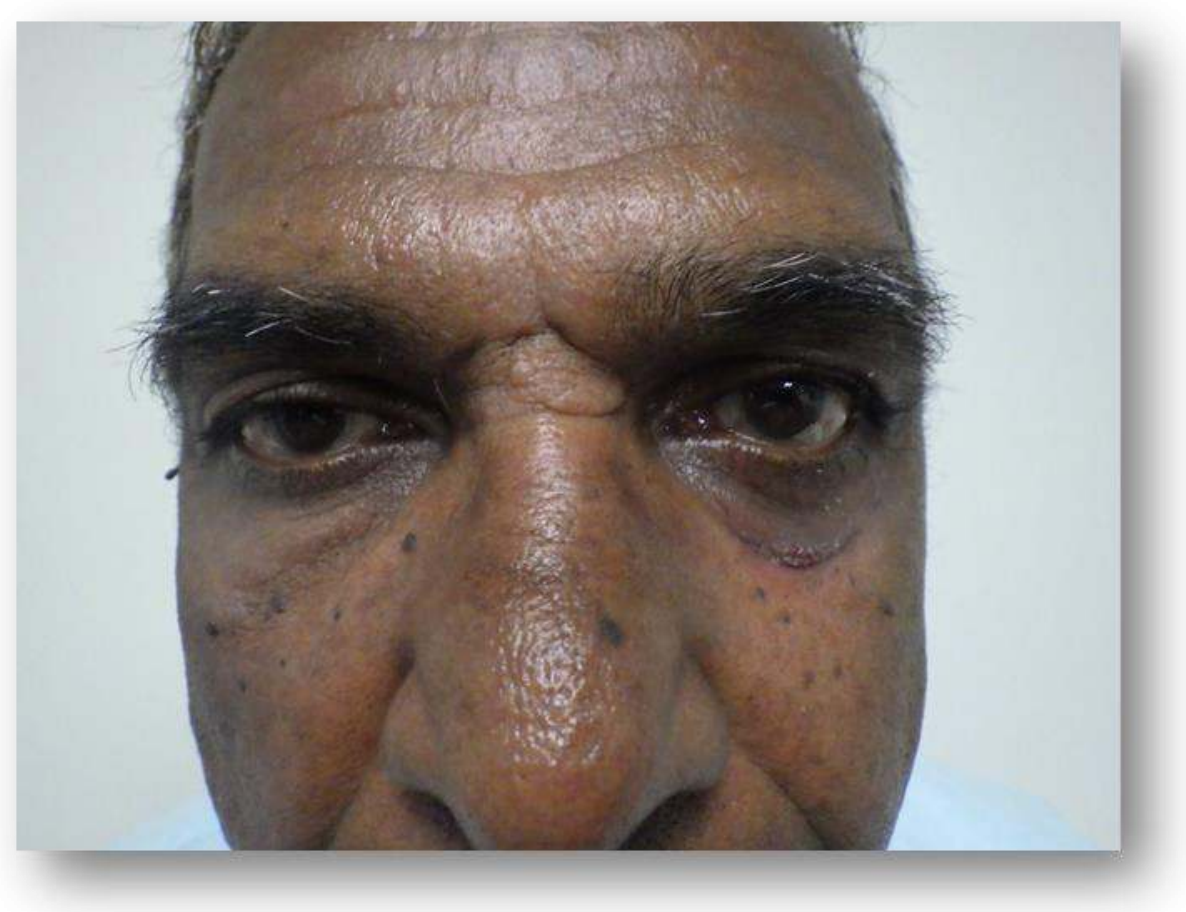

Copyright @ 2017 Author Names and Contact Details 